\title{
VLSI Supply Chain Security Risks and Mitigation Techniques: A Survey
}

\author{
Bao Liu ${ }^{1}$, Gang $\mathrm{Qu}^{2}$
}

\begin{abstract}
Hardware is the foundation of security and trust for any security system. However, recent study has revealed that hardware is subject to a number of security risks. Some of the most severe risks come from the VLSI supply chain. Such risks compromise the foundation of any existing security design. In this paper, we present a systematic survey on these security risks and their corresponding mitigation techniques.
\end{abstract}

Keywords: Security; VLSI; IP Theft; Hardware Trojan.

\section{Introduction}

2 A security system is implemented in many lay3 ers. Cryptographic algorithms, including symmetric ci${ }_{4}$ phers, public-key ciphers, and hash functions, form a 5 set of primitives that can be used as building blocks to ${ }_{6}$ construct security mechanisms that target specific ob7 jectives, such as confidentiality, integrity, and availabil8 ity [1]. Rigorous theoretical analysis and design of 9 cryptosystems and security protocols are achieved only 10 based on certain assumptions of low level implemen11 tation. For example, it is typically assumed that im12 plementations of cryptographic computations are ideal ${ }_{13}$ "black boxes" whose internals can neither be observed 14 nor interfered with by any malicious entity. Specifically, 15 all the existing cryptographic primitives have proofs of ${ }_{16}$ security based on two assumptions: (1) read-proof hard17 ware; that is, hardware that prevents an adversary from 18 reading anything about the information stored within it; 19 and (2) tamper-proof hardware; that is, hardware that ${ }_{20}$ prevents an adversary from changing anything in the ${ }_{21}$ information stored within it. However, these assump22 tions are far from reality. Almost all known security ${ }_{23}$ attacks on embedded systems target the implementation ${ }_{24}$ rather than taking on the computational complexity of 25 breaking a cryptographic primitive employed in a se${ }_{26}$ curity mechanism [2]. An interesting analogy can be ${ }_{27}$ drawn in this regard between a strong cryptographic al${ }_{28}$ gorithm and a highly secure lock on the front door of a

\footnotetext{
${ }^{1}$ B. Liu is with the University of Texas at San Antonio, San Antonio, TX 78249.

${ }^{2} \mathrm{G}$. Qu is with the University of Maryland, College Park, MD 20742.

Preprint submitted to Computers $\mathcal{E}$ Graphics
}

${ }_{29}$ house. Burglars attempting to break into a house will so rarely try all the combinations necessary to pick such a ${ }_{31}$ lock; they may break in through windows, break a door ${ }_{32}$ at its hinges, or rob the owner of a key as they are trying ${ }_{33}$ to enter the house [3].

${ }_{34}$ Further, there is a growing trend in recent years to 35 migrate software-based security solutions to hardware${ }_{36}$ based security solutions for much enhanced resistance ${ }_{37}$ to software-based security attacks. Such systems range ${ }_{38}$ from smartcards to specialized secure co-processing 39 boxes, wherein hardware provides the source of secu40 rity and trust, e.g., concealing confidential data and pro${ }_{41}$ viding trustworthy computation for a number of secu${ }_{42}$ rity primitives, e.g., platform identification and authen${ }_{43}$ tication, identity, key and certificate management, low${ }_{44}$ level cryptography, I/O access control, safe data storage, 45 and code integrity checking. Famous examples include ${ }_{46}$ Trusted Platform Module (TPM) [4], ARM TrustZone ${ }_{47}$ [5], Microsoft Next Generation Secure Computing Base ${ }_{48}$ [6], and academic secure processors such as XOM [7], ${ }_{49}$ CODESSEAL [8], AEGIS [9, 10], REM [11], SP [12] ${ }_{50}$ and SPEF [13, 14].

${ }_{51}$ All these security solutions are based on the as52 sumption that hardware is trustworthy in possessing ${ }_{53}$ all the desired security properties. However, hardware ${ }_{54}$ is subject to a variety of security risks as recent re55 search has revealed, which compromises the founda${ }_{56}$ tion of all the existing security designs. In this paper, ${ }_{57}$ we present a systematic review on the security risks ${ }_{58}$ in a VLSI supply chain and their respective state-of${ }_{59}$ the-art mitigation techniques. We do not cover rela${ }_{60}$ tively well studied areas of physical access-based at${ }_{61}$ tacks such as side-channel analysis $[15,16]$ and fault ${ }_{62}$ injection $[17,18,19]$. We further focus on Application-

February 15, 2016 


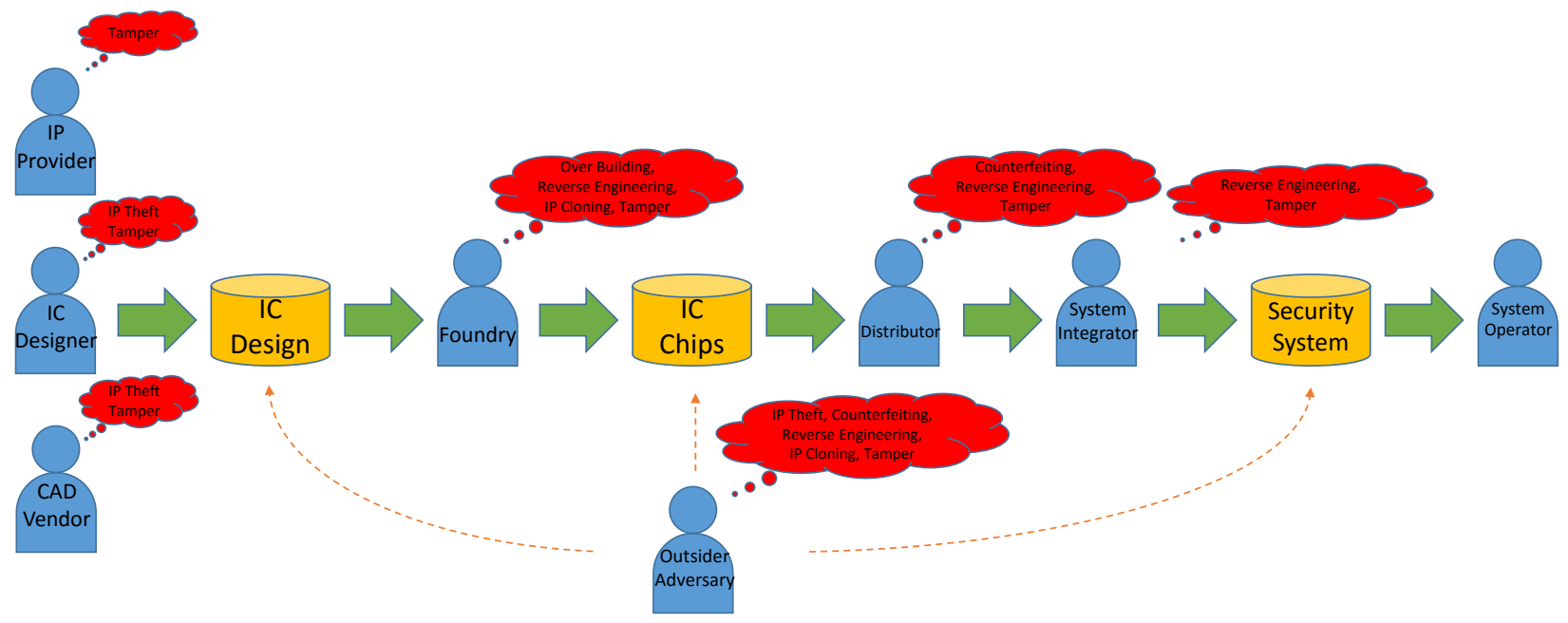

Figure 1: VLSI supply chain security risks.

${ }_{63}$ Specific Integrated Circuit (ASIC) security, while inter64 ested readers may refer to existing literature on FPGA 65 security [20, 21, 22, 23].

66 The rest of this paper is organized as follows. We ${ }_{67}$ present an overview on VLSI supply chain security risks 68 in Section 2 and their state-of-the-art mitigation tech69 niques in Sections 3 and 4. We conclude in Section 6.

\section{2. VLSI Supply Chain Security Risks}

71 Today's semiconductor industry involves multiple 72 business entities on a global scale in design, manu${ }_{73}$ facturing, system integration and distribution of VLSI 74 chips and systems. Without an effective security mech75 anism, a rogue element in this process - such as an ${ }_{76}$ IP provider, an IC design house, a CAD company, a 77 foundry, a distributor or a system integrator - can eas78 ily steal design IPs or tamper with an IC design; there 79 is also a possibility that an outsider adversary steals de${ }_{80}$ sign IPs or tampers with the design (Fig. 1). We catego${ }_{81}$ rize such security risks into two groups: (1) IP theft and 82 misuse, where an adversary obtains an IP through an il${ }_{83}$ legal channel or uses the authentic IPs illegally, and (2) ${ }_{84}$ IC tamper, where an adversary modifies the functional${ }_{85}$ ity, performance or other features of an IC for various ${ }_{86}$ malicious purposes. In terms of the security objectives ${ }_{87}$ we mentioned in the introduction, IP theft and misuse 8s compromise IP confidentiality; while IC tamper com89 promises IC design authenticity and integrity. In the 90 rest of this section, we will elaborate security risks from ${ }_{91}$ each of the two categories.

\section{${ }_{92}$ 2.1. IP Theft and Misuse}

93 IC designs and the intellectual properties (IPs) cre${ }_{94}$ ated during the design process can be protected legally 95 through the means such as patent, copyright, trademark, ${ }_{96}$ and trade secret. Design IPs (such as Verilog code, de97 sign data, and FPGA configuration bitstream files) can 98 also be encrypted to prevent illegal copy or misuse. ${ }_{99}$ However, IP theft is an easy and very profitable busi100 ness practice due to the lack of effective law enforce101 ment mechanisms, and the need of keeping IP easy to 102 use and reuse. Evidently, we have seen rampant IP theft 103 and misuse in semiconductor industry in recent years. ${ }_{104}$ For example, in over-building, a contract manufacturer 105 fills an order and continues to build more chips and sell 106 them [23]. In cloning, a competitor makes a copy of 107 a design by stealing part or all of a system's intellec108 tual property (IP) [23]. In reverse engineering, a com109 petitor extracts not only all the IPs from a design, but 110 also explicit details on how the design works - by pack111 age removal, delayering, imaging, circuit extraction and 112 analysis - which allows the IPs to be reused, improved, ${ }_{113}$ or disguised to thwart possible legal actions $[23,24] .^{3}$

114 One common feature of such attacks is that rogue 115 business entities are driven by profit. They are interested 116 in IP theft or misuse rather than IP or IC tamper. For ex117 ample, an IP licensee may misuse an IP for designs that 118 are not included in the license agreement. This leads 119 to financial loss for the IP owner without necessarily

\footnotetext{
${ }^{3}$ Despite its potential application in IP theft and IC tampering, reverse engineering is a legal practice, e.g., to collect competitor intelligence, determine patent infringements, and detect hardware Trojans [25].
} 
120 compromising the authentic design and the end product. ${ }_{121}$ Furthermore, profit-driven attacks such as over-building 122 and cloning often happen at a large scale.

\section{2.2. IC Tamper}

${ }_{124}$ The end products of IP theft and misuse are often 125 known as counterfeits, which are work-alike or cloned ${ }_{126}$ products with illegal use of a brand name. Such coun${ }_{127}$ terfeits are widespread; the United States Department 128 of Defense has identified more than one million suspect 129 counterfeit parts associated with supply chain compro130 mises in two years [26]. Such counterfeit chips may be ${ }_{131}$ made from recycled chips of degraded lifetime, reliabil${ }_{132}$ ity or performance. The most severe form of hardware ${ }_{133}$ security risks is that on such counterfeit chips an adver${ }_{134}$ sary may tamper with the genuine design and install a ${ }_{135}$ "Trojan horse" component which once triggered acts as ${ }_{136}$ a logic bomb or information leak back door [27]. An ${ }_{137}$ entire Trojan program may be hidden in hardware, e.g., ${ }_{138}$ in a Trojan ROM beside a processor (Figure 4) [28]. An ${ }_{139}$ adversary may launch such an attack from a foundry, ${ }_{140}$ from a system assembly line, or, anyone who captures ${ }_{141}$ a hardware device may replace a genuine chip with a 142 counterfeit chip on a printed-circuit board (PCB). A 143 tampered system may still function as expected for min144 imum footprint, except that it provides a hidden attack 145 mechanism for knowledgeable attackers. Such IC tam${ }_{146}$ per attacks may evade all the existing security solutions 147 implemented at higher (e.g., software application or op${ }_{148}$ erating system) levels. For example, the existing static 149 and dynamic code integrity verification techniques de150 tect tamper in the file system, memory or stack rather 151 than a hardware Trojan $[7,9,10,12,29,30]$. As a re152 sult, IC tamper attacks compromise a fundamental as${ }_{153}$ sumption of the existing security system designs which 154 is the trustworthiness of hardware. They request serious 155 rethinking on security system design.

156 IC tamper attacks may not lead to obvious profit, 157 while hidden incentives cannot be ruled out, since pos${ }_{158}$ sible attackers such as amateur hackers, criminal orga159 nizations and nation states have different resources, ca160 pacities and incentives. In some cases, IC tamper can be 161 an economically viable practice, for example, installing ${ }_{162}$ data-collecting hardware spyware. Due to the potential ${ }_{163}$ severity of such attacks and the limitations of the exist164 ing countermeasure techniques, the Comprehensive Na165 tional Cyber Security Initiative has identified this supply ${ }_{166}$ chain risk management problem as a top national prior167 ity [31].

${ }_{168}$ The existing VLSI design and verification techniques 169 are insufficient in mitigating such security risks and en170 suring hardware design authenticity, integrity and con-
Table 1: VLSI supply chain security risks and mitigation techniques.

\begin{tabular}{|l|l|}
\hline Security Risks & Mitigation Techniques \\
\hline Reverse Engineering & Obfuscation \\
\hline Over-Building & $\begin{array}{l}\text { Watermarking, Fingerprinting, } \\
\text { Metering }\end{array}$ \\
\hline Counterfeiting & Fingerprinting, Metering \\
\hline Cloning & Watermarking, Fingerprinting \\
\hline Tamper (IC Design) & $\begin{array}{l}\text { Simulation, Formal Verification, } \\
\text { Detection, Obfuscation }\end{array}$ \\
\hline Tamper (IC Chip) & $\begin{array}{l}\text { Reverse Engineering, } \\
\text { Testing, Side Channel Analysis, } \\
\text { Design for Tamper Detection } \\
\text { Design for Tamper Prevention }\end{array}$ \\
\hline
\end{tabular}

171 fidentiality. In today's IC industry, once an IC design is 172 delivered to a foundry, the designers have no effective 173 mechanism to prevent IP theft and misuse. The existing 174 testing techniques only verify if an IC design meets all 175 the specifications. They do not detect the presence of 176 any extra functionality which may be a security back177 door. Table 1 summarizes the VLSI supply chain secu178 rity risks that we know today and their respective state179 of-the-art mitigation techniques. We detail each of these 180 techniques as follows.

\section{${ }_{181}$ 3. Techniques Against IP Theft and Misuse}

\section{3.1. Design Obfuscation}

183 Obfuscation is a long-standing problem in computer ${ }_{184}$ security and cryptography as a potentially very power185 ful tool against reverse engineering. The classic "black186 box" definition of obfuscation is to create an implemen${ }_{187}$ tation of a function $f$ that reveals nothing about $f$ except 188 its input-output behavior. Intuitively, a circuit obfus${ }_{189}$ cator $O$ is an efficient algorithm that, given a circuit $C$ 190 implementing some function $f$, outputs another circuit ${ }_{191} O(C)$ such that (i) (preserving functionality) it computes 192 (perhaps approximately) the same function as $f$, (ii) ${ }_{193}$ (polynomial slowdown) its size is bounded by a given ${ }_{194}$ polynomial $p$ in the size of the original circuit, i.e., ${ }_{195}|O(C)| \leq p(|C|)$, and, (iii) ("virtual black-box" property) 196 for any efficient adversary that computes some predicate 197 on $O(C)$, there exists an efficient simulator that com198 putes the same predicate with black-box access to an 199 oracle that evaluates $f[32,33,34]$.

200 Recent theoretical studies have shown that, (1) there 201 exist functions that cannot be obfuscated, and (2) there 202 exist functions that can be obfuscated. Barak et al. have 203 shown the existence of (contrived) classes of functions 204 which are not obfuscatable, or, a general purpose obfus205 cator does not exist [32]. The only positive obfuscation 206 result is of point functions, which are Boolean functions 
207 that return logic one on exactly one input, for example, a 208 password checker program. Canetti and Wee separately 209 showed how to obfuscate a point function based on a 210 random oracle, e.g., a hash function that hides all details $211[33,35]$. An obfuscated point function queries the ran212 dom oracle on an input, and compares the answer with a 213 stored value. For example, a password checker program ${ }_{214}$ encrypts an input, and compares the encryption result 215 with a stored value, which is an encrypted password. ${ }_{216}$ As a result, it achieves the virtual black box property of 217 obfuscation. This scheme is based on a weaker defini${ }_{218}$ tion of obfuscation, which says that there is a negligi${ }_{219}$ ble probability to distinguish an adversary circuit based 220 on the obfuscated scheme and a simulator based on a 221 black box of the function. As a result, this obfuscation 222 scheme of point functions cannot be extended to obfus${ }_{223}$ cate arbitrary Boolean functions [34], except some spe224 cific classes of functions such as d-CNFs [36].

225 VLSI obfuscation is achievable based on certain spe226 cial manufacturing technologies, such as split manufac227 turing, 3D IC integration or embedded reconfigurable 228 logic in ASIC design, which realize the aforementioned 229 "black-box" property. The presence of obfuscated mod230 ules does not guarantee that the entire design is obfus${ }_{231}$ cated, as the adversary may still gain knowledge on or 232 tamper with the un-obfuscated part of the design. De233 sign obfuscation methods may not stop an adversary, ${ }_{234}$ but certainly increase their cost of reverse engineering. ${ }_{235}$ To conclude our discussion on obfuscation, we list rep${ }_{236}$ resentative recently proposed circuit obfuscation tech237 niques.

- In split manufacturing, while the logic gates and the interconnects at the lower metal layers are mass produced at an untrusted foundry, the interconnects at the higher metal layers are customized at a later stage at a trusted site in a semi-customized IC manufacturing technology [37]. An adversary at an untrusted foundry has only black-box access to the upper-layer interconnects. However, an adversary may reconstruct the upper-layer interconnects and the whole design based on subgraph isomorphism [38], and the complexity of doing so can be further reduced based on certain VLSI design objectives, constraints and rules, e.g., minimum wirelength, no combinational loop, and no simultaneous multiple driver of any logic signal [39].

- In 3D IC integration, among several stacked dies mounted on an interposer, a trusted die can be manufactured at a trusted foundry while the other dies and the interposer may be manufactured at an

Table 2: Comparison of IC design obfuscation techniques by attack resistance and hardware cost.

\begin{tabular}{|l|l|l|}
\hline Obfuscation Techniques & Attack Resistance & Hardware Cost \\
\hline Split Manufacturing & Low & Low \\
\hline 3D IC & High & Medium \\
\hline Reconfigurable Logic & High & High \\
\hline IC Camouflaging & Low & Medium \\
\hline Logic Locking & Low & Low \\
\hline
\end{tabular}
205 resistance (the computation complexity of the problem

untrusted foundry. An adversary at the untrusted foundry has only black-box access to a trusted die. A trusted die contains logic gates besides interconnects. As a result, the complexity for an adversary to re-construct the whole design is much higher than split manufacturing.

- Similarly, certain modules of an ASIC design can be realized in reconfigurable logic. Such reconfigurable logic can be constructed by a customer or system engineer after the manufacturing and supply process. As a result, any supply chain adversary has only black-box access to it $[40,41]$.

- In IC camouflaging, multiple logic gates are fabricated in identical or similar layout patterns [42, 43]. While even without high resolution microscopy equipment, an adversary can re-construct a logic netlist including camouflaged logic gates at certain computation complexity $[44,45]$.

- In logic encryption or logic locking, a combinational logic network is augmented by a group of XOR/XNOR logic gates [46, 47], multiplexers combining different logic cones [48], LUTs forming a reconfigurable logic barrier [46], or permuting the logic inputs/outputs [49], such that only applying a specific vector to the augmented inputs leads to the correct logic. Similarly, an FSM can be augmented by a group of extra finite states which form an obfuscated mode, such that only a correct sequence of inputs transit the FSM out of the obfuscated mode and set the FSM to the correct initial state in the normal operation mode $[50,51,52,48,53]$. These techniques prevent an adversary from unlawful operation of a device. However, with knowledge on the function of a protected module, e.g., from the design of the rest of the system, an adversary can recover the key, e.g., based on IC testing techniques [40, 41, 54].

Table 3 compares these technqiues in terms of attack 295 resistance (the computation complexity of the problem 


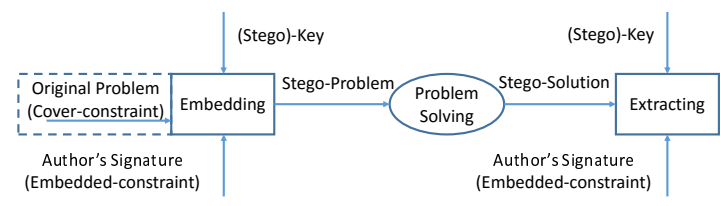

Figure 2: Constraint-based IC watermark embedding and extraction

296 that an attacker must solve to recover the authentic de297 sign) and hardware cost.

\section{3.2. Digital Watermarking}

299 IP and IC watermarking is to secretly convey the in300 formation on content ownership and IP/IC rights. Com${ }_{301}$ pared with steganography, IP and IC watermarking fur302 ther requires the property of robustness, i.e., being in303 feasible to remove or make useless without destroying 304 the IP/IC at the same time. Watermarking has been 305 applied to protect IPs in all forms, including Verilog 306 codes [55], combinational logic [56, 57], sequential cir307 cuits [58], finite state machines [59] and FPGA designs ${ }_{308}^{30}$ [60], physical design [61], and CAD tools [62]. A sur309 vey can be found [63].

310 Digital watermarking has been widely used for iden311 tification, annotation, and copyright of multimedia data 312 such as text, image, audio, and video. Traditional wa313 termarking techniques take advantage of the limitation 314 of human visual and auditory system and embed a sig315 nature to an original data set as minute errors. This 316 actually changes the original data and cannot be di317 rectly used for the protection of hardware design IPs 318 because the value of design IP relies on its correct func${ }_{319}$ tionality and performance. To solve the problem of 320 embedding digital watermark into IC without chang321 ing its functionality, a novel constraint-manipulation322 based methodology was developed in the late 1990's in ${ }_{323}$ UCLA. It was first reported in a series of papers in 1998 ${ }_{324}[64,56,61,65,66,67]$ and most of the early results can 325 be found in a monograph published in 2003 [68].

${ }_{326}$ A constraint-based watermarking technique trans327 lates the to-be-embedded signatures into a set of ad328 ditional design constraints during the design and im${ }_{329}$ plementation of an IP to uniquely encode the signa330 tures into the IP (Figure 2). To hide a signature, the ${ }_{331}$ designer first creates another set of constraints using 332 his secret key. These constraints are selected in such ${ }_{333}$ a way that they do not conflict with the constraints in 334 the original design. Then the original and additional 335 constraints are combined to form an over-constrained 336 stego-problem. The stego-problem, instead of the orig${ }_{337}$ inal problem, is solved to obtain a stego-solution which 338 has the designer's digital watermark embedded.
339 During the design and optimization process, the de${ }_{340}$ sign with these watermarks will have certain specific ${ }_{341}$ properties such as constraints. These properties can 342 be extracted from the final design as the proof to the ${ }_{343}$ designer's ownership, and the designer can regener344 ate them using his signature together with his secret 345 key. Cryptography functions such as one-way hash and ${ }_{346}$ stream cipher will be used to generate the embedded347 constraints. To facilitate the detection of watermarks, a ${ }_{348}$ public watermarking scheme was proposed in [69].

349 Hardware IP watermarking techniques can be catego350 rized as static and dynamic $[60,63,70]$. In static hard351 ware IP watermarking, the watermark is detected with352 out running the IP. The dominant techniques are based 353 on including extra constraints which indicate ownership 354 information in solving an optimization problem [69], 355 such as logic optimization [57], place and route [61]. In 356 dynamic hardware IP watermarking, the watermark can 357 only be detected by running the IP. For example, water358 marks can be embedded in logic don't care conditions 359 [55], a watermarked FSM gives the encrypted owner360 ship information for a given input vector sequence [71], ${ }_{361}$ or, exhibits a unique property for the input vector se${ }_{362}$ quence which is the encrypted ownership information ${ }_{363}[58]$.

\section{3.3. IC Fingerprinting}

365 IC watermarking embeds a designer's signature into 366 an IC to claim his ownership and IP/IC rights against 367 IP/IC piracy. Such watermarks do not help forensics 368 such as tracing a copyright violator who distributed il369 legal copies. The digital fingerprinting techniques solve ${ }_{370}$ this problem by embedding a buyer's signature along ${ }_{371}$ with a designer's watermark in an IC design. Both the 372 watermark and the fingerprint are invisible identifiers 373 that are embedded in the design permanently for the pur374 pose of IP protection. All copies of an IP share an iden375 tical watermark, while each copy of an IP has a unique 376 fingerprint.

377 Any fingerprinting technique has to address two fun${ }_{378}$ damental problems: (i) how to generate IPs with unique 379 fingerprints effectively and (ii) how to distribute these 380 fingerprinted IPs to the users. While the problem of ${ }_{381}$ distributing fingerprinted copies is similar to the well382 studied problem of distributing other artifacts such as звз multimedia data, there are several unique challenges 384 in the IP generation problem: How to create a large 385 amount of copies with no duplicated fingerprints? How 386 to keep the overhead or IP quality degradation at min${ }_{387}$ imum? How to minimize the time and complexity of ${ }_{388}$ generating multiple fingerprinted IPs (ideally keeping it 389 close to that of designing a single copy)? 


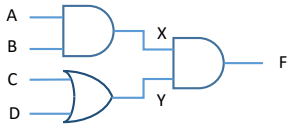

(a)

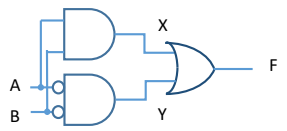

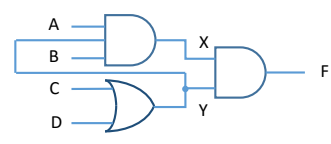

(b)

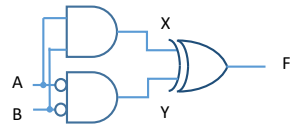

(d)
Figure 3: Digital fingerprinting by creating logic equivalent IPs. (a) and (b) differ in an interconnect. (c) and (d) differ in a logic gate with a don't care input vector $(x=y=1)$.

390 Because of these difficulties, there are much less IC 391 fingerprinting techniques than IC watermarking tech392 niques in literature. Caldwell et al. pioneered in creat393 ing fingerprints based on specific VLSI CAD optimiza394 tion heuristics [70]. They demonstrated IC fingerprint395 ing taking as examples some of the VLSI design related ${ }_{396}$ NP-hard problems such as partitioning, Boolean satis397 fiability (SAT), graph coloring and standard-cell place398 ment problems. These problems are solved by iterative 399 optimization techniques, such that specific constraints 400 similar to those in watermarking can be introduced in 401 iterations to create many unique solutions.

402 A conceptually-different fingerprinting approach for ${ }_{403}$ the graph coloring problem is proposed in [72], where 404 the authors effectively add new constraints to a graph by 405 either adding new edges to create cliques or introduc406 ing new nodes and edges to duplicate existing nodes. ${ }_{407}$ This increases the solution space such that solving the 408 problem once leads to creation of multiple unique fin409 gerprints. A major issue with this approach and that in ${ }_{410}[70]$ is that these techniques must be implemented in an ${ }_{411}$ early stage of VLSI design. This leads to significant 412 increase in design time and cost. For instance, each fin${ }_{413}$ gerprinted IC must have a different mask, which is im${ }_{414}$ practical given the cost of masks.

415 Recently reported are two practical fingerprinting ${ }_{416}$ methods based on the observability don't cares (ODC) 417 and satisfiability don't cares (SDC) in logic design 418 [73, 74]. For example, insertion of an extra intercon419 nect in Fig. 3 (a) leads to Fig. 3 (b) without changing 420 the Boolean logic function at Y due to the ODC condi${ }_{421}$ tion [73]; while replacing the OR gate in Fig. 3 (c) by 422 an XOR gate leads to Fig. 3 (d) under a SDC condition ${ }_{423}$ [74]. Such changes can be made after IC fabrication, for ${ }_{424}$ example, based on reconfigurable logic.

\section{3.4. IC Metering}

426 IC metering targets another critical security problem ${ }_{427}$ in IC supply chain: with the asymmetric relationship 428 between an IC design house and an foundry, once a de429 sign house delivers a design to a foundry, the design 430 house will have no control on what the foundry can do 431 to the design. This creates the risk of IC over-building 432 wherein an extra volume of chips are fabricated without 433 the design house's permission. "IC metering is a set of ${ }_{434}$ security protocols that enable a design house to achieve 435 post-fabrication control over their ICs" [75].

${ }_{436}$ The basic concepts behind IC metering is to embed a ${ }_{437}$ unique tag to each IC and make sure that the tag is under ${ }_{438}$ the control of the design house instead of the foundry. ${ }_{439}$ Many different types of tags have been proposed and 440 used for hardware metering. They can be categorized 441 based on various criteria.

442 In passive metering, a tag can only be used for chip ${ }_{443}$ identification. In active metering, a tag can further en444 able, disable, or control a chip. Active metering meth445 ods can be further classified as internally controlled 446 and external controlled based on whether the control 447 is part of the design. Intrinsic metering does not need 448 any help from additional components or design mod449 ification. Extrinsic metering methods do. Depending 450 whether the tag interacts with the chip's functionality, 451 we have non-functional metering and functional meter452 ing. Finally, some tags can be reproduced and some 453 cannot which are known as unclonable tags.

${ }_{454}$ The serial number technique is one of the most popu455 lar and earliest device tagging technique. A serial num456 ber can be physically indented on the device or stored ${ }_{457}$ permanently in the memory. These tags are passive, ex458 trinsic, non-functional, and reproducible. The fact that 459 such tags can be reproduced makes it unsuitable to pre460 vent IC over-building.

461 The ICID tag technique was first proposed in 2007 462 [76]. In this technique, a sequence of control signals se463 lect an array of transistors to drive a capacitive load. The 464 output voltage differs for each chip due to inherent IC 465 manufacturing process variations. Because such vari466 ations include random and uncontrollable components, ${ }_{467}$ An ICID is considered an unclonable tag and thus can 468 be applied against IC over-building.

469 The ICID tag technique is the first scheme for gen470 erating a weak PUF or random chip ID based on pro471 cess variations [77]. Other PUF schemes include ar472 biter, ring oscillator, and SRAM-based [78, 77]. Essen473 tially, a PUF is an (at least partly) disordered physical ${ }_{474}$ system $P$ that can be challenged with so-called exter475 nal stimuli or challenges $c$, upon which it reacts with 
Table 3: Comparison of IC watermarking, fingerprinting and metering techniques by attack resistance, design, verification and hardware costs.

\begin{tabular}{|l|l|l|l|l|}
\hline $\begin{array}{l}\text { IP-Protecting } \\
\text { Techniques }\end{array}$ & $\begin{array}{l}\text { Attack } \\
\text { Resistance }\end{array}$ & $\begin{array}{l}\text { Design } \\
\text { Cost }\end{array}$ & $\begin{array}{l}\text { Verification } \\
\text { Cost }\end{array}$ & $\begin{array}{l}\text { Hardware } \\
\text { Cost }\end{array}$ \\
\hline IC Watermarking & High & Low & Low/High & Low \\
\hline IC Fingerprinting & High & High & Low/High & Medium \\
\hline IC Metering & Low & Low & Medium & Low \\
\hline
\end{tabular}

${ }_{476}$ corresponding responses $r$. Contrary to standard dig477 ital systems, these responses depend on the micro- or 478 nanoscale structural disorder of the PUF. It is assumed 479 that this disorder cannot be cloned or reproduced ex480 actly, not even by the PUF's original manufacturer, and ${ }_{481}$ that it is unique to each PUF. Any PUF $P$ thus imple482 ments a unique and individual function $f_{P}$ that maps ${ }_{483}$ challenges $c$ to responses $r=f_{P}(c)[77,79]$. Such a 484 response can be exploited for deriving a standard dig485 ital key that is not stored in the hardware and hard to 486 extract, for system identification, or for more complex ${ }_{487}$ cryptographic protocols such as oblivious transfer (OT), 488 bit commitment (BC), or key exchange (KE) [77, 79]. A ${ }_{489}$ PUF needs to achieve uniqueness, randomness and reli490 ability [80, 78].

\section{3.5. Comparison}

492 Table 3 compares IC watermarking, fingerprinting 493 and metering technqiues. IC watermarks and finger494 prints are integrated in IC design, such that an attacker 495 needs to reverse engineer and understand an IC design 496 to remove or forge a watermark or fingerprint. As a 497 result, they have high attack resistance. IC watermark498 ing techniques based on extra design constraints or cir499 cuitry have a little design and hardware cost. The cost 500 of IC fingerprinting techniques are higher than IC wa501 termarking techniques because a lot more IC finger502 prints are needed for each customer. Dynamic IC wa503 termarking/fingerprinting techniques are easy to verify, 504 while static IC watermarking/fingerprinting techniques 505 require reverse engieering and have a higher cost. IC 506 metering techniques depend on a ICID tag or PUF that 507 is separate from the IC design. As a result, the design 508 and hardware costs for a RFID tag or PUF only count 509 for a small percentage of that of a whole chip. How510 ever, their attack resistances need to be examined case 511 by case. For example, Rührmair et al. discussed many 512 assumptions and limitations of PUF-based techniques in 513 the context of different security protocols $[77,79]$.

\section{${ }_{514}$ 4. Techniques Against IC Tamper}

515 We have two groups of IC tamper detection tech516 niques. The first group of techniques detect tamper for a ${ }_{517}$ given IC design. The second group of techniques detect 518 tamper for a given IC chip. We will discuss IC tamper 519 prevention at last.

\section{4.1. IC Design Tamper Detection}

${ }_{521}$ This group of techniques address the following prob522 lem.

${ }_{523}$ Problem 1 (IC Design Tamper Detection). Given an ${ }_{524}$ IC design (e.g., RTL design in form of Boolean logic 525 expression) and its implementation (e.g., logic design in ${ }_{526}$ form of gate-level netlist or layout design), verify that ${ }_{527}$ the implementation faithfully realizes the design with528 out any additional functionality.

${ }_{529}$ A number of existing techniques address this prob${ }_{530}$ lem, including Layout Versus Schematic (LVS), formal ${ }_{531}$ verification and simulation. However, they do not guar${ }_{532}$ antee hardware Trojan detection, and ongoing research ${ }_{533}$ is producing new techniques. We elaborate as follow.

\section{${ }_{534}$ 4.1.1. Simulation}

535 Simulation is one of the mainstream IC design verifi${ }_{536}$ cation techniques. However, there are a number of lim${ }_{537}$ itations in applying simulation techniques for hardware ${ }_{538}$ tamper detection. The existing simulation techniques 539 verify an IC design against its specifications; they do not 540 target IC tamper detection or extra functionality identi${ }_{541}$ fication. A hardware Trojan may perform an extra task ${ }_{542}$ without tampering the authentic functionalities. Further, ${ }_{543}$ a hardware Trojan may be triggered by a rare event such 544 as power glitch or IC aging which may not even be mod545 eled in a digital system simulation environment. With${ }_{546}$ out a priori knowledge, the likelihood is minimal for a ${ }_{547}$ simulator to trigger and detect a hardware Trojan.

\section{4.1.2. Formal Verification}

549 Formal verification verifies if an implementation con550 forms to its specification by a formal (e.g., mathemati$\left.{ }_{551} \mathrm{cal}\right)$ method [81]. This includes equivalence checking 552 and property checking, e.g., of security requirements 553 such as absence of unprotected path from confidential 554 data. Equivalence checking determines if an implemen${ }_{555}$ tation realizes no more and no less than what is spec${ }_{556}$ ified. The "no more" part is exactly needed for Tro${ }_{557}$ jan detection. The existing LVS techniques check the 
${ }_{558}$ equivalence between an IC layout and its schematic de559 sign, e.g., based on graph isomorphism. For logic equiv560 alence between a Boolean logic expression and a gate${ }_{561}$ level netlist, one can represent both in a canonical form, 562 e.g., Ordered Binary Decision Diagram (OBDD), and ${ }_{563}$ check the graph isomorphism of the two OBDDs [82]. ${ }_{564}$ The OBDD technique achieves a polynomial average 565 runtime for the NP-complete problem which worst case ${ }_{566}$ runtime remains exponential. However, the complexity 567 of checking functional equivalence of sequential sys568 tems remains very high: two functional equivalent se569 quential systems may look very different due to retim570 ing optimization and/or different finite state encodings; 571 while the exponential number of state transition paths 572 leads to the state explosion problem. To mitigate this 573 problem, for a finite state machine, one may represent 574 (1) each finite state in a vector of Boolean variables, (2) 575 all the finite states in a Boolean function which returns 576 true for all the finite state representations in Boolean 577 variable vectors, and (3) all the state transitions $x R y$ in 578 a Boolean function with two sets of Boolean variables, 579 one for state $x$ and the other for state $y$. The OBDD 580 technique can be subsequently applied for equivalence ${ }_{581}$ and property checkings [83]. Such techniques are in the 582 category of symbolic model checking which consist of 583 systematically exhaustive exploration of a mathematical 584 model based on smart and domain-specific abstraction 585 techniques. Symbolic model checking techniques are 586 more scalable than explicit-state model checking tech587 niques which enumerate each reachable state. However, 588 their scalability is still limited.

589 Another category of formal verification techniques 590 are deductive verification. This usually involves de591 scribing the subject system and the properties to verify 592 in one of the interactive or automatic theorem provers 593 such as HOL [84], Coq [85], PVS [86], etc. Notable ${ }_{594}$ examples include the four color theorem proof which 595 was based on Coq [87]. Recent techniques include 596 the Proof-Carrying Code (PCC) technique wherein soft597 ware developer/vendors provide proofs for customer598 specified safety policies in a binary executable [88], and 599 the similar Proof-Carrying Hardware (PCH) framework 600 which is a SAT solver-based combinational equivalence 601 checker between a design specification and a design im602 plementation on a reconfigurable platform [89, 90], and 603 a new PCH framework which uses the Coq functional 604 language [85] for proof construction and leverages the ${ }_{605}$ Coq platform for automatic proof validation [91, 92]. 606 These techniques require that the verification engineer 607 have detailed understanding on the system and the prop608 erties to verify and convey them in formal specification.
609 4.1.3. Redundant Logic and Hard-to-Excite Signal $610 \quad$ Identification

611 Even if an implementation is logic equivalent to its 612 original design, a hardware Trojan may still be hidden ${ }_{613}$ in redundant logic, and could be activated by fault injec614 tion, e.g., based on perturbation of power supply, clock, 615 or injection of an optical fault [19] or an IC aging sensor ${ }_{616}[28,93]$. To address this problem, techniques such as ${ }_{617}$ Unused Circuit Identification (UCI) have been proposed 618 [94] and improved [95]. Further, ATPG techniques can 619 be leveraged to identify redundant or untestable logic 620 [96]. Because hardware Trojans are supposed to be trig621 gered by a rare event, another group of techniques locate 622 hard-to-excite signals as candidates of hardware Trojan 623 trigger [97]. These techniques can be combined. For ${ }_{624}$ example, Banga and Hsiao proposed a four-step proce625 dure to locate suspicious logic in third-party IPs: (1) A 626 sequential ATPG technique removes easy-to-detect sig627 nals. (2) A full-scan N-detect ATPG technique identi628 fies hard-to-excite and/or propagate signals. (3) To nar629 row down the list of suspected signals and identify the ${ }_{630}$ gates associated with a hardware Trojan, a SAT solver ${ }_{631}$ checks equivalence of the suspicious netlist containing ${ }_{632}$ the rarely triggered signals against the netlist of the cir${ }_{633}$ cuit exhibiting correct behavior. (4) Finally, clusters of ${ }_{634}$ untestable gates in the circuit were determined using the 635 region isolation approach on the suspected signals list ${ }_{636}$ [96]. Zhang and Tehranipoor proposed another multi${ }_{637}$ stage approach which includes assertion based verifica638 tion, code coverage analysis, redundant circuit removal, 639 equivalence analysis and use of sequential Automatic 640 Test Pattern Generation (ATPG) for suspicious signals 641 identification [98]. These techniques do not need an au642 thentic design as reference. However, these techniques ${ }_{643}$ are limited as a hardware Trojan may not be based on 644 redundant logic or a hard-to-excite signal.

\section{4.2. IC Chip Tamper Detection}

646 This group of techniques address the following prob647 lem.

${ }_{648}$ Problem 2 (IC Chip Tamper Detection). Given an ${ }_{649} I C$ design and an IC chip, verify that the IC chip ${ }_{650}$ faithfully realizes the design without any additional 651 functionality.

\section{4.2.1. Reverse Engineering}

653 Part of the technical difficulty of the IC chip tamper 654 detection problem is that a verification engineer may not 655 even know the design details of a chip. Reverse engi656 neering can be applied to extract the design details of 
657 a chip, such that the IC design tamper detection tech658 niques in subsection 4.1 can be applied. This method 659 can detect any tamper by a designer, an IP provider, a ${ }_{660} \mathrm{CAD}$ vendor, a system integrator or a distributor. How${ }_{661}$ ever, an adversary at an untrusted foundry may tamper ${ }_{662}$ with only a few IC chips, while the existing reverse en${ }_{663}$ gineering techniques are destructive: traditional IC re664 verse engineering techniques require decapsulation and ${ }_{665}$ passive layer removal, while new techniques such as X${ }_{666}$ ray microscopy damage transistors [24]. As a result, ${ }_{667}$ combination of reverse engineering and IC design tam668 per detection techniques cannot be applied to all the ${ }_{669}$ chips and cannot guarantee detection of hardware tam670 per by an adversary at a foundry.

\section{4.2.2. Testing}

672 To detect a hardware Trojan by testing, (1) the testing ${ }_{673}$ procedure must activate the hardware Trojan, and (2) the 674 activated hardware Trojan leads to a behavior deviation 675 of the VLSI system such as an incorrect output that can 676 be observed. However, neither is easy to achieve. Ac677 tivating a hardware Trojan is very difficult since a hard678 ware Trojan can be triggered by a rare event which is 679 unknown to a test engineer $[99,100,101]$. If the hard${ }_{680}$ ware Trojan trigger logic includes an IC aging sensor, ${ }_{681}$ the hardware Trojan cannot be activated before the IC ${ }_{682}$ is sufficiently aged $[28,93]$. Even if a hardware Trojan ${ }_{683}$ is activated, the hardware Trojan may still keep a min684 imum footprint, for example, sending out confidential 685 information in a side channel [102] or by steganography 686 [103] without tampering with the result of any authentic 687 computation in the host system.

\section{4.2.3. Side Channel Analysis}

${ }_{689}$ Besides IC testing techniques, side channel analysis 690 techniques have been proposed for IC tamper detection. 691 These techniques collect IC characterizations in a side 692 channel such as timing performance [104], power con${ }_{693}$ sumption [105], temperature, or electromagnetic emis694 sion [106], and find outliers for candidates of tampered ${ }_{695}$ chips. These techniques rely on a golden tamper-free 696 reference design which may be achieved by reverse en697 gineering a few chips [106] or by self referencing [107]. ${ }_{698}$ However, a few significant problems exist: (1) the sig699 nificant effect of parametric variations could easily bury 700 the effect of a small hardware Trojan; and (2) it is very 701 difficult to activate a hardware Trojan. Without be702 ing activated, a dormant hardware Trojan has very little 703 footprint, e.g., possibly in leakage [108]. These make 704 side channel analysis very difficult.

\section{4.2.4. IC Design for Tamper Detection}

706 Due to the limitations in IC testing and side chan707 nel analysis, IC design techniques are needed to facil708 itate tamper detection. For example, ring oscillator709 based on-chip sensors are proposed to detect hardware 710 Trojan-induced power supply voltage droop [109, 110]. 711 The built-in self-authentication (BISA) technique lever712 ages the existing built-in self-test (BIST) techniques 713 [109, 111].

714 Another group of techniques are based on concurrent 715 checking. A variety of concurrent checking techniques 716 are available in the traditional fault-tolerant computing 717 literature $[112,113]$. These techniques are appealing for 718 tamper detection because they do not require hardware 719 Trojan activation which is difficult to achieve without a 720 priori knowledge on the hardware Trojan. On the other 721 hand, such techniques are tamper detection but not tam722 per prevention techniques.

723 In concurrent checking, a hardware system generates 724 information bits and check bits, e.g., parity bits, du725 plicate of the information bits as in a dual-module re726 dundancy (DMR) scheme, or in a more efficient error${ }_{727}$ detecting code (EDC) $[112,113]$. Checking the consis728 tency between the information bits and the check bits 729 detects runtime errors such as soft errors or adversary 730 tampers (e.g., triggered by a timer) which cannot be de731 tected by testing.

${ }_{732}$ At system level, fault tolerant processor design in${ }_{733}$ cludes a variety of redundant execution and concurrent ${ }_{734}$ checking techniques [113]. (1) Lockstepping schemes 735 compare internal states (e.g., program control flow [114, 736 115, 116], hardware control signals [117], memory ac737 cess [118], and reasonableness of results $[119,120])$ in ${ }_{738}$ each cycle with duplicated program runs in a watchdog 739 co-processor. (2) Redundant Multi-Threading (RMT) 740 schemes compare only outputs of committed instruc741 tions [121, 122, 123, 124, 125]. (3) EDCC-based 742 hardware assertion techniques lead to more hardware743 efficient fault tolerant processors compared with lock744 stepping or RMT [126, 127, 128].

745 Concurrent checking techniques have been adopted 746 for tamper detection. For example, the TrustNet 747 and DataWatch architectures include on-chip monitors 748 which check the consistency of control signals and data 749 in a microprocessor [129]. Against remote attacks and 750 physical attacks, e.g., wherein an adversary has physi751 cal access to the hardware and can tamper with memory 752 busses or instructions and data stored in memory chips, 753 the DEFENSE architecture includes a FPGA which per754 forms runtime concurrent integrity checking besides en755 cryption and decryption for instruction and data blocks 


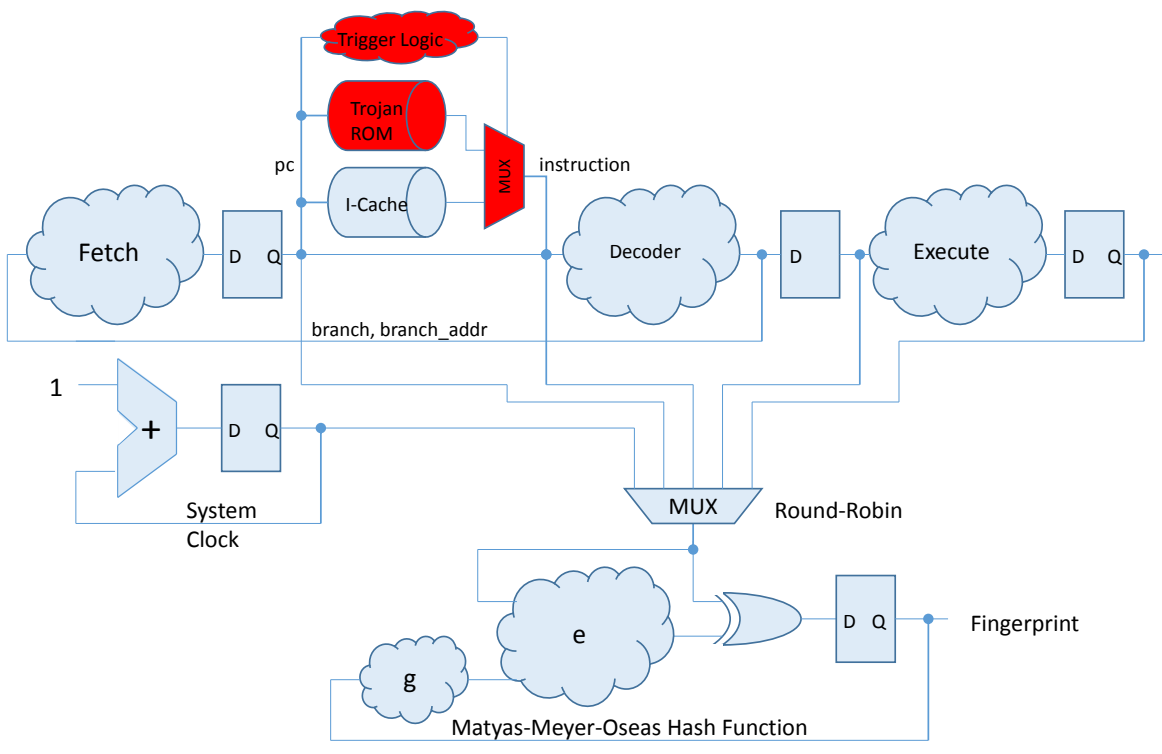

Figure 4: A code injection hardware Trojan including a Trojan ROM, multiplexers, and trigger logic (red), and a tamper-evident architecture including multiplexers that sample runtime signals including the system time in a round-robin scheme, and a fingerprint generator based on the Matyas-Meyer-Oseas hash function (below the instruction pipeline) in a processor.

756 between a processor core and a memory chip [130]. ${ }_{757}$ Fetch-time or runtime integrity checking is included in 758 many secure processors such as AEGIS [9, 10], REM 759 [11], SP [12], and SPEF [13, 14] against remote attacks 760 wherein an adversary performs code injection, reuse or ${ }_{761}$ data injection or substitution attacks via a communica${ }_{762}$ tion channel. Further against IC tamper attacks such ${ }_{763}$ as code injection from a hardware Trojan (Fig. 4 (a)), 764 concurrent checking needs to be applied against run765 time signals inside an IC chip, and such a checking 766 mechanism needs to be protected from tamper by a sup${ }_{767}$ ply chain adversary, e.g., based on reconfigurable logic, 768 split manufacturing, or reconfigurable resistive RAM 769 (RRAM) switches [131]. For example, the Tamper770 Evident Architecture (TEA) computes a fingerprint or 771 keyed cryptographic hash for runtime signals during the 772 computation in a hardware system, and verify such a fin${ }_{773}$ gerprint off-chip for computation integrity verification 774 and malicious program detection (Fig. 4). As a result, 775 a supply chain adversary or hardware Trojan (1) can776 not generate correct check bits or fingerprint for a ma777 licious program, and (2) cannot tamper with the check778 ing mechanism [28]. This technique verifies integrity 779 and authenticity of a program run without guarantee780 ing the integrity and authenticity of the system, e.g., it ${ }_{781}$ does not detect a dormant hardware Trojan. The cost of 782 such techniques can be controlled similarly to the exist783 ing Design for Testability (DFT) techniques [132].

\section{4.3. IC Tamper Prevention}

785 Besides tamper detection, we further need tamper re786 sponse (recovery or self-destruction) and tamper evi787 dence (recording and digital forensics) techniques [20]. ${ }_{788} \mathrm{~A}$ harder problem is tamper prevention. A number of 789 techniques have been proposed for tamper prevention 790 with limited effectiveness.

791 A straightforward solution is IC design obfuscation. 792 However as we know obfuscation of an entire VLSI sys793 tem is not possible while some modules may be obfus794 cated such as based on reconfigurable logic or a trusted 795 die $[40,41]$.

796 Another technique is to obfuscate data or runtime sig797 nals in a hardware system for data confidentiality and 798 tamper prevention. Instruction and data encryption for 799 storage is a common technique, while their decryption 800 brings performance cost $[7,8]$. Bus scrambling such as 801 by permutation or XORing with a pseudo-random num802 ber achieves only weak cryptographic strength [101], 803 while achieving stronger cryptographic strength comes 804 with significant cost. Private circuit techniques address 805 the problem of achieving data confidentiality in the 806 presence of an attacker who can observe at most $t$ sig807 nals in a hardware system at any given time [133, 134]. ${ }_{808}$ Fascinating progress has been achieved in the field of 809 homomorphic cryptography [135] and secure multi810 party computation [136, 137], allowing arbitrary com${ }_{811}$ putation based on encrypted data - albeit at a prohibitive 812 cost for efficient VLSI application. 


\section{${ }_{813}$ 5. Hardware Security Research Trends}

814 Hardware security is under heated research. Ongoing 815 research development is leading to rapid innovations. 816 We notice several trends in this field: (1) VLSI tech817 nology development has made some traditional tech818 niques such as side channel analysis increasingly dif${ }_{819}$ ficult (higher integration leads to a decreasing signal-to820 noise ratio in cutting-edge technologies for side channel 821 analysis). While this also provides opportunities to de822 velop new security solutions based on emerging VLSI 823 technologies, for example, for Truly Random Num824 ber Generation (TRNG) and PUFs. (2) New research 825 trends in system integration such as Internet of Things ${ }_{826}$ and Cyber-Physical Systems demand security research 827 for such emerging systems. IoT/CPS are complex sys828 tems including software, firmware and hardware com829 ponents. They are expected to be deployed in diverse, 830 dynamic, and potentially hostile environment, such as, ${ }_{831}$ for example, an adversary may easily gain physical pos832 session of an IoT/CPS device, and launch hardware at833 tacks. The traditional security research addresses secu834 rity protocols, primitives, and their software implemen835 tations, while hardware is assumed to be trustworthy. 836 This research gap gives rise to recent hardware security 837 research efforts. (3) From a system perspective, new 838 hardware or system security research works need to be 839 based on more realistic attack models which include as 840 many as possible attack methods. (4) Examining hard841 ware security solutions in the context of higher level 842 security primitives and protocols such as in $[77,79]$ ${ }_{843}$ provides new perspectives. (5) Examining security so844 lutions from an economic and/or social perspective is 845 much needed to facilitate security solution deployment 846 in the real world because the cost and benefit of security 847 techniques are ultimately shared by parties in a supply 848 chain, an industry ecosystem, and a society. Security849 oriented business management and policy making and 850 enhancement mechanisms are much needed.

\section{${ }_{851}$ 6. Summary}

852 Hardware security risks such as from a VLSI supply 853 chain come under scrutiny only recently. Such security 854 risks compromise the foundation of all existing secu855 rity designs. Consequently, research on their mitiga856 tion techniques has been intensive in recent years. In 857 this paper, we present a systematic survey on the hard858 ware security risks from a VLSI supply chain and their 859 state-of-the-art countermeasure techniques. Although 860 significant progress has been made over the years, many ${ }_{861}$ important problems remain open and critical solutions
862 missing in this field. We hope that this survey help in${ }_{863}$ crease public awareness to the problem and foster fur864 ther technology development in the field.

\section{${ }_{865}$ Acknowledgements}

866 The authors thank NSF support by grant CISE${ }_{867} 1538418$, and anonymous reviewers for their comments.

\section{${ }_{868}$ Reference}

1] B. Schneier, Applied Cryptography: Protocols, Algorithms and Source Code in C, John Wiley and Sons, 1996.

[2] S. Ravi, A. Raghunathan, S. Chakradhar, Tamper resistance mechanisms for secure embedded systems, in: Intl. Conf. on VLSI Design, 2004

[3] B. Schneier, Security Pitfalls in Cryptography, http://www.schneier.com/essay-pitfalls.html.

[4] Trusted Computing Group, Trusted Platform Module (TPM) Specifications.

URL http: //www . trustedcomputinggroup.org/ resources/tpm_main_specification

[5] ARM, Building a secure system using trustzone technology, ARM Limited.

[6] Microsoft, Next-generation secure computing base URL http: //www . microsoft . com/resources/ngscb/ default.mspx

[7] D. Lie, C. Thekkath, M. Mitchell, et al., Architecture support for copy and tamper resistant software, in: Proc. International Conference on Architecture Support for Programming Languages and Operating Systems (ASPLOS-IX), 2000, pp 168-177.

[8] O. Gelbart, P. Ott, B. Narahari, R. Simha, A. Choudhary, J. Zambreno, Codesseal: Compiler/fpga approach to secure applications, in: Proc. IEEE Int. Conf. on Intelligence and Security Informatics, 2005, pp. 530-535.

[9] G. E. Suh, D. Clarke, B. Gassend, M. van Dijk, S. Devadas, AEGIS: architecture for tamper-evident and tamper-resistant processing, in: Proc. International Conference on Supercomputing, 2003

[10] G. E. Suh, C. W. O’Donnell, I. Sachdev, S. Devadas, Design and implementation of a single-chip secure processor using physical random functions, in: Proc. International Symposium on Computer Architecture, 2004.

[11] A. M. Fiskiran, R. B. Lee, Runtime execution monitoring (REM) to detect and prevent malicious code execution, in: Proc. IEEE Intl. Conf. Computer Design, 2004.

[12] R. B. Lee, P. C. S. Kwan, J. P. McGregor, J. Dwoskin, Z. Wang, Architecture for protecting critical secrets in microprocessors, in: Proc. International Symposium on Computer Architecture (ISCA), 2005, pp. 2-13.

[13] D. Kirovski, M. Drinic, M. Potkonjak, Enabling trusted software integrity, in: Proc. 10th Int. Conf. Architecture Support for Programming Languages and Operating Systems, 2002, pp. $108-120$.

[14] M. Drinic, D. Kirovski, A hardware-software platform for intrusion prevention, in: Porc. 37th Ann. IEEE/ACM Intl. Symp. Microarchitecture, 2004, pp. 233-242.

[15] P. C. Kocher, Timing attacks on implementations of DiffieHellman, RSA, DSS, and other systems, Advances in Cryptology - CRYPTO'96, Lecture Notes in Computer Science V. 1109 (1996) 104-113. 
[16] P. Kocher, J. Jaffe, B. Jun, Differential power analysis, in: Proc. Intl. Cryptography Conf. Advances in Cryptography, 1999, pp. 388-397.

[17] H. Choukri, M. Tunstall, Handbook of Information Security, Volume 3, John Wiley and Sons, 2006.

[18] S. Skorobogatov, R. Anderson, Optical fault induction attacks, in: Proc. Cryptographic Hardware and Embedded Systems, 2003, pp. 2-12.

19] J. G. J. van Woudenberg, M. F. Witteman, F. Menarini, Practical optical fault injection on secure microcontrollers, in: Proceedings of the 2011 Workshop on Fault Diagnosis and Tolerance in Cryptography, 2011, pp. 91-99.

20] Altera, Anti-tamper capabilities in FPGA designs. URL http: //www . altera.com/literature/wp/ wp-01066-anti-tamper-capabilities-fpga.pdf/

[21] S. Drimer, Volatile FPGA design security - a survey (2008). URL http: //www . saardrimer . com/sd410/papers / fpga_security.pdf

22] Microsemi, Overview of data security using microsemi FPGAs and SoC FPGAs. URL http: //www .microsemi.com/document-portal/ doc_view/132873-overview-of-data-securityusing-microsemi-fpgas-and-soc-fpgas

[23] Microsemi, Overview of design security using microsemi FPGAs and SoC FPGAs.

URL http: //www .microsemi.com/document-portal/ doc_view/132862-overview-of-design-securityusing-microsemi-fpgas-and-soc-fpgas

[24] R. Torrance, D. James, The state-of-the-art in IC reverse engineering, in: Cryptographic Hardware and Embedded Systems CHES 2009, Vol. 5747 of Lecture Notes in Computer Science, 2009, pp. 363-381.

[25] Chipworks. [link]. URL http: //www . chipworks . com/

[26] U. S. Senate, Inquiry into counterfeit electronic parts in the department of defense supply chain, report of the committee on armed services (May 21, 2012).

[27] M. Beaumont, B. Hopkins, T. Newby, Hardware trojans prevention, detection, countermeasures (a literature review), DSTO-TN-1012, unclassified, Tech. rep., Australian Government, Department of Defense, Defense Science and Technology Organization, http://www.dtic.mil/get-tr-doc/pdf?AD=ADA547668 (2011).

[28] B. Liu, R. Sandhu, Fingerprint-based detection and diagnosis of malicious programs in hardware, IEEE Trans. on Reliability.

[29] R. Elbaz, D. Champagne, C. Gebotys, R. B. Lee, N. Potlapally, L. Torres, Hardware mechanisms for memory authentication: A survey of existing techniques and engines, Trans. on Comput. Sci. (2009) 1-22.

[30] R. B. Lee, D. K. Karig, J. P. Mcgregor, Z. Shi, Enlisting hardware architecture to thwart malicious code injection, in: In Proceedings of the 2003 International Conference on Security in Pervasive Computing, 2003, pp. 237-252.

[31] National Security Council, The Comprehensive National Cybersecurity Initiative. URL http://www . whitehouse.gov/cybersecurity/ comprehensive-national-cybersecurity-initiative

[32] B. Barak, O. Goldreich, R. Impagliazzo, S. Rudich, A. Sahai, S. Vadhan, K. Yang, On the (im)possibility of obfuscating programs, in: Proc. International Conference on Cryptography, 2001, pp. 1-18.

[33] R. Canetti, Towards realizing random oracles: Hash functions that hide all partial information, in: Proc. International Conference on Cryptography, 1997, pp. 455-469.
[34] A. Narayanan, V. Shmatikov, On the limits of point function obfuscation (2006) URL http: //eprint.iacr . org/2006/182

[35] H. Wee, On obfuscating point functions, in: Proc. ACM Symp. the Theory of Computing, 2005.

[36] Z. Brakerski, G. N. Rothblum, Black-box obfuscation for dcnfs, Cryptography ePrint Archieve (2013). URL http: //eprint.iacr .org/2013/557

[37] IARPA, Trusted integrated chips (TIC), http://www.iarpa.gov/index.php/research-programs/ tic/baa (2011).

[38] F. Imeson, A. Emtenan, S. Garg, M. V. Tripunitara, Securing computer hardware using $3 \mathrm{~d}$ integrated circuit (ic) technology and split manufacturing for obfuscation, in: Proc. 22nd USENIX Security Symposium, 2013, pp. 495-510.

[39] J. Rajendran, O. Sinanoglu, R. Karri, Is split manufacturing secure, in: Proc. Conference on Design Automation and Test in Europe, 2013, pp. 1259 - 1264.

[40] B. Liu, B. Wang, Embedded reconfigurable logic for ASIC design obfuscation against supply chain attacks, in: Proc. Conference on Design Automation and Test in Europe, 2014.

[41] B. Liu, B. Wang, Reconfiguration-based vlsi design for security, IEEE Journal on Emerging and Selected Topics in Circuits and Systems.

[42] L. W. Chow, J. P. Baukus, B. J. Wang, R. P. Cocchi, Camouflaging a standard cell based integrated circuit, uS Patent 8,151,235 (2012).

URL http://www.google.com/patents/US8151235

[43] SypherMedia, Circuit camouflage technology: SMI IP protection and anti-tamper technologies (2012).

URL http://www.smi.tv/ SMI_SypherMedia_Library_Intro.pdf

[44] M. E. Massad, S. Garg, M. V. Tripunitara, Integrated circuit (IC) decamouflaging: Reverse engineering camouflaged ICs within minutes, in: Proc. of NDSS, 2015.

[45] J. Rajendran, M. Sam, O. Sinanoglu, R. Karri, Security analysis of integrated circuit camouflaging, in: ACM Conference on Computer and Communications Security, 2013, pp. 709-720.

[46] A. Baumgarten, A. Tyagi, J. Zambreno, Preventing IC piracy using reconfigurable logic barriers, IEEE Design and Test of Computers (2010) $66-75$.

[47] J. Roy, F. Koushanfar, I. Markov, EPIC: Ending piracy of integrated circuits, in: Proc. Conference on Design Automation and Test in Europe, 2008, pp. 1069 - 1074.

[48] R. S. Chakraborty, S. Bhunia, HARPOON: an obfuscationbased SoC design methodology for hardware protection, IEEE Trans. Computer-Aided Design 28(10) (2009) 1493-1502.

[49] J. A. Roy, F. Koushanfar, I. L. Markov, Protecting bus-based hardware IP by secret sharing, in: Proc. ACM/IEEE Design Automation Conf., 2008, pp. 846-851.

[50] Y. M. Alkabani, F. Koushanfar, Active hardware metering for intellectual property protection and security, in: Proc. USENIX Security Symposium, 2007, pp. $291-306$.

[51] R. S. Chakraborty, S. Bhunia, Hardware protection and authentication through netlist level obfuscation, in: Proc. IEEE Intl. Conf. Computer-Aided Design, 2008, pp. 674-677.

[52] R. S. Chakraborty, S. Bhunia, Security against hardware Trojan through a novel application of design obfuscation, in: Proc. IEEE Intl. Conf. Computer-Aided Design, 2009, pp. 113-116.

[53] A. R. Desai, M. S. Hsiao, et al., Interlocking obfuscation for anti-tamper hardware, in: CSIIRW, 2012, pp. 1 - 4.

[54] J. Rajendran, Y. Pino, O. Sinanoglu, R. Karri, Security analysis of logic obfuscation, in: Proc. ACM/IEEE Design Automation Conf., 2012, pp. $83-89$.

[55] L. Yuan, R. Pari, G. Qu, Soft IP protection: Watermarking 
HDL source codes, in: 6th Information Hiding Workshop, 2004, pp. 224-238.

[56] A. B. Kahng, J. Lach, W. H. Mangione-Smith, S. Mantik, I. L. Markov, M. Potkonjak, P. Tucker, H. Wang, G. Wolfe, Watermarking techniques for intellectual property protection, in: Proc. ACM/IEEE Design Automation Conf., 1998.

[57] A. B. Kahng, J. Lach, W. H. Mangione-Smith, S. Mantik, I. L. Markov, M. Potkonjak, P. Tucker, H. Wang, G. Wolfe, Constraint-based watermarking techniques for design intellectual property protection, IEEE Trans. Computer-Aided Design 20(10) (2001) 1236-1252.

[58] A. L. Oliverira, Robust techniques for watermarking sequential circuit designs, in: Proc. ACM/IEEE Design Automation Conf., 1999, pp. 837-842.

[59] L. Yuan, G. Qu, Information hiding in finite state machine, in: 6th Information Hiding Workshop, 2004, pp. 340-354.

[60] A. K. Jain, L. Yuan, P. R. Pari, G. Qu, Zero overhead watermarking technique for fpga designs, in: Proc. Great Lakes Symp. VLSI, 2003, pp. 147-152.

[61] A. B. Kahng, S. Mantik, I. L. Markov, M. Potkonjak, P. Tucker, H. Wang, G. Wolfe, Robust ip watermarking methodologies for physical design, in: Proc. ACM/IEEE Design Automation Conf., 1998, pp. 782-787.

[62] L. Yuan, G. Qu, A. Srivastava, VLSI CAD tool protection by birthmarking design solutions, in: Proc. Great Lakes Symp. VLSI, 2005, pp. 341-344.

[63] A. T. Abdel-Hamid, S. Tahar, M. Aboulhamid, A survey on IP watermarking techniques, Design Automation for Embedded Systems 9 (2004) 211-227.

[64] J. Lach, W. H. Mangione-Smith, M. Potkonjak, Fingerprinting digital circuits on programmable hardware, in: Information Hiding Workshop, 1998, pp. 16-31.

[65] J. Lach, W. H. Mangione-Smith, M. Potkonjak, Fpga fingerprinting techniques for protecting intellectual property, in: Custom Integrated Circuits Conference, 1998, pp. 299-302.

[66] J. Lach, W. H. Mangione-Smith, M. Potkonjak, Signature hiding techniques for fpga intellectual property protection, in: Proc. IEEE Intl. Conf. Computer-Aided Design, 1998, pp. 186-189.

[67] G. Qu, M. Potkonjak, Analysis of watermarking techniques for graph coloring problem, in: Proc. IEEE Intl. Conf. ComputerAided Design, 1998, pp. 190-193.

[68] G. Qu, M. Potkonjak, Intellectual property protection in VLSI designs: theory and practice, Springer Science \& Business Media, 2003.

[69] G. Qu, Publicly detectable watermarking for intellectual property authentication in vlsi design, IEEE Trans. ComputerAided Design 21(11) (2002) 1363-1368.

[70] A. E. Caldwell, H.-J. Choi, A. B. Kahng, S. Mantik, M. Potkonjak, G. Qu, J. L. Wong, Effective iterative techniques for fingerprinting design ip, IEEE Trans. Computer-Aided Design 23(2) (2004) 208-215.

[71] I. Torunoglu, E. Charbon, Watermarking-based copyright protection of sequential functions, IEEE J. Solid State Circuits 35(3) (2000) 434-440.

[72] G. Qu, M. Potkonjak, Fingerprinting intellectual property using constraint-addition, in: Design Automation Conference, 2000 .

[73] C. Dunbar, G. Qu, A practical circuit fingerprinting method utilizing observability dont care conditions, in: Proc. ACM/IEEE Design Automation Conf., 2015, pp. 113-118.

[74] C. Dunbar, G. Qu, Satisfiability don't care condition based circuit fingerprinting techniques, in: Proc. Asian and South $\mathrm{Pa}-$ cific Design Automation Conference, 2015.

[75] F. Koushanfar, Hardware metering: A survey, in: M. Tehra- nipoor, C. Wang (Eds.), Introduction to Hardware Security and Trust, Springer New York, 2012, pp. 103-122.

76] K. Lofstrom, W. R. Daasch, D. Taylor, IC identification circuit using device mismatch, in: Proc. IEEE Solid State Circuits Conference, 2000, pp. 372-373.

77] U. Rührmair, S. Devadas, F. Koushanfar, Security based on physical unclonability and disorder, in: M. Tehranipoor, C. Wang (Eds.), Introduction to Hardware Security and Trust, Springer New York, 2012, pp. 65-102.

[78] C. Böhm, M. Hofer, Physical Unclonable Functions in Theory and Practice, 1st Edition, Springer International Publishing, 2014

[79] U. Rührmair, M. van Dijk, PUFs in security protocols: Attack models and security evaluations, in: IEEE Symposium on Security and Privacy, 2013, pp. 286-300.

[80] M. Bhargava, Reliable, secure, efficient physical unclonable functions, Ph.D. thesis, Carnegie Mellon University (2013).

[81] McFarland, Formal verification of sequential hardware, IEEE Trans. Computer-Aided Design 12(5) (1993) 633-654.

[82] R. E. Bryant, Graph-based algorithms for boolean function manipulation, IEEE Trans. Computers C-35(8) (1986) 677 - 691

[83] K. L. McMillan, Symbolic model checking: An approach to the state explosion problem, Ph.D. thesis, Carnegie Mellon University (1992).

[84] H. Community, HOL interactive theorem prover, https://hol-theorem-prover.org/.

[85] INRIA, The coq proof assistant, http://coq.inria.fr/ (2010).

[86] SRI, PVS specification and verification system, http://pvs.csi.sri.com/ (2014).

[87] G. Gonthier, Formal proof - the four-color theorem, Notices of the American Mathematical Society 55(11) (2008) 1382 1392.

[88] G. C. Necula, Proof-carrying code, in: POPL '97: Proceedings of the 24th ACM SIGPLAN-SIGACT Symposium on Principles of Programming Languages, 1997, pp. 106-119.

[89] S. Drzevitzky, U. Kastens, M. Platzner, Proof-carrying hardware: Towards runtime verification of reconfigurable modules, in: International Conference on Reconfigurable Computing and FPGAs, 2009, pp. 189-194.

[90] S. Drzevitzky, Proof-carrying hardware: Runtime formal verification for secure dynamic reconfiguration, in: Field Programmable Logic and Applications (FPL), 2010 International Conference on, 2010, pp. 255-258.

[91] E. Love, Y. Jin, Y. Makris, Enhancing security via provably trustworthy hardware intellectual property, in: HardwareOriented Security and Trust (HOST), 2011 IEEE International Symposium on, 2011, pp. 12-17.

[92] E. Love, Y. Jin, Y. Makris, Proof-carrying hardware intellectual property: A pathway to trusted module acquisition, IEEE Transactions on Information Forensics and Security 7 (1) (2012) 25-40.

[93] Y. Shiyanovskii, F. Wolff, C. Papachristou, D. Weyer, W. Clay, Exploiting semiconductor properties for hardware trojans, ePrint arXiv:0906.3834 (2009). URL http://arxiv.org/pdf/0906.3834

[94] M. Hicks, M. Finnicum, S. T. King, M. M. K. Martin, J. M. Smith, Overcoming an untrusted computing base: Detecting and removing malicious hardware automatically, in: Proc. of IEEE Symposium on Security and Privacy, 2010, pp. 159-172.

[95] C. Sturton, M. Hicks, D. Wagner, S. T. King, Defeating uci: Building stealthy and malicious hardware, in: Proc. of IEEE Symposium on Security and Privacy, 2011, pp. 64-77.

[96] M. Banga, M. Hsiao, Trusted RTL: Trojan detection methodology in pre-silicon designs, in: IEEE International Symposium 
1235 [114] D. Lu, Watchdog processors and structural integrity checking, 1236 1237 [115] M. Namjoo, Techniques for concurrent testing of vlsi processor 1238

1239 [116] J. P. Shen, M. A. Schuette, On-line self-monitoring using sig1240 natured instruction streams, in: Proc. IEEE Intl. Test Conf., $1241 \quad$ 1983, pp. 275-282.

1242 [117] S. F. Daniels, A concurrent test technique for standard micro1243 processors, in: Dig. Papers Compcon Spring 83, 1983, pp. 1244 389-394.
1245 [118] M. Namjoo, E. J. McCluskey, Watchdog processors and capa1246 bility checking, in: Dig. Papers 12th Annu. Int. Symp. Fault 1247 Tolerant Comput., FTCS-12, 1982, pp. 245-248.

1248 [119] A. Mahmood, E. J. McCluskey, Concurrent error detection us1249 ing watchdog processors - a survey, IEEE Trans. Computers $1250 \quad 37(2)$ (1988) 160-174.

1251 [120] P. P. Shirvani, E. J. McCluskey, Fault-tolerant systems in a 1252 space environment: The CRC ARGOS project, in: CRC Tech1253 nical Report No. 98-2 (CSL TR No. 98-774), 1998.

1254 [121] T. M. Austin, Diva: A reliable substrate for deep submicron 1255 microarchitecture design, in: Proc. Annu. Intl. Symp. on Mi1256 croarchitecture (MICRO), 1999, pp. 196-207.

257 [122] D. Bernick, B. Bruckert, P. D. Vigna, D. Garcia, R. Jardine, 1258 J. Klecka, J. Smullen, NonStop advanced architecture, in: 1259 Proc. Intl. Conf. on Dependable Systems and Networks(DSN), $1260 \quad 2005$, pp. 12-21.

261 [123] M. A. Gomaa, C. Scarbrough, T. N. Vijaykumar, I. Pomeranz, 1262 Transient fault-recovery for chip multiprocessors, in: Proc. In1263 ternational Symposium on Computer Architecture, 2003, pp. $1264 \quad 98-109$

265 [124] E. Rotenberg, Ar-smt: A microarchitectural approach to fault 1266 tolerance in microprocessors, in: Annu. Fault-Tolerant Com1267 puting Systems (FTCS), 1999, p. 84.

1268 [125] T. N. Vijaykumar, I. Pomeranz, K. Cheng, Transient fault re1269 covery using simultaneous multithreading, in: Proc. Interna1270 tional Symposium on Computer Architecture, 2002.

1271 [126] T. J. Slegel, R. M. Averill, M. A. Check, B. C. Giamei, B. W. 1272 Krumm, C. A. Krygowski, W. H. Li, J. S. Liptay, J. D. Mac1273 Dougall, T. J. McPherson, J. A. Navaroo, E. M. Schwarz, 1274 K. Shum, C. F. Web, IBM's S/390 G5 microprocessor design, 1275 IEEE Micro (1999) 12-23.

1276 [127] L. Spainhower, T. A. Gregg, IBM S/390 parallel enterprise 1277 server G5 fault tolerance: A historical perspective, IBM Jour1278 nal of Research and Development 43(5/6) (1999) 863-873.

1279 [128] C. Webb, z6 - the next-generation mainframe microprocessor 1280 (2007).

1281 URL http://speleotrove.com/decimal/ 1282 IBM-z6-mainframe-microprocessor-Webb.pdf

1283 [129] A. Waksman, S. Sethumadhavan, Tamper evident microproces1284 sors, in: Proc. IEEE Symp. on Security and Privacy, 2010, pp. $1285 \quad 1-16$

286 [130] M. Abramovici, P. Bradley, Integrated circuit security - new 1287 threats and solutions, in: Proc. of the 5th Annual Workshop on 1288 Cyber Security and Information Intelligence Research: Cyber 1289 Security and Information Intelligence Challenges and Strate1290 gies, 2009.

1291 [131] S. Mitra, H.-S. P. Wong, S. Wong, The Trojan-proof chip, IEEE $1292 \quad$ Spectrum 2 (2015) $46-51$

1293 [132] M. L. Bushnell, V. D. Agrawal, Essentials of Electronic Test1294 ing For Digital, Memory, And Mixed-Signal VLSI Circuits, $1295 \quad$ Norwell, MA: Kluwer, 2000.

1296 [133] Y. Ishai, A. Sahai, D. Wagner, Private circuits: Securing hard1297 ware against probing attacks, in: Proc. International Confer1298 ence on Cryptography, 2003, pp. 463-481.

1299 [134] Y. Ishai, M. Prabhakaran, A. Sahai, D. Wagner, Private cir$1300 \quad$ cuits ii: Keeping secrets in tamperable circuits, in: Advances in Cryptology - EUROCRYPT 2006, 25th Annual International Conference on the Theory and Applications of Cryptographic Techniques, Vol. 4004 of Lecture Notes in Computer Science, Springer, 2006, pp. 308-327. URL http://www . iacr .org/cryptodb/archive/2006/ EUROCRYPT/2508/2508.pdf

[135] C. Gentry, A fully homomorphic encryption scheme, Ph.D. thesis, Stanford University (2009). URL http: //crypto.stanford.edu/craig/ 
1310 [136] C. Orlandi, Is multiparty computatoin any good in practice?, in: ICASSP, 2011.

1312 [137] J. Saia, M. Zamani, Recent results in scalable multi-party com1313 putation, in: G. Italiano, T. Margaria-Steffen, J. Pokorny, J.1314 J. Quisquater, R. Wattenhofer (Eds.), SOFSEM 2015: Theory 1315 and Practice of Computer Science, Vol. 8939 of Lecture Notes 1316 in Computer Science, Springer Berlin Heidelberg, 2015, pp. $1317 \quad 24-44$. 